\title{
ÓLEO ESSENCIAL DE ERVA-CIDREIRA SOBRE FITOPATÓGENOS DE ESPÉCIES FLORESTAIS E AGRÍCOLAS
}

\author{
Bruna Cristine Martins de Sousa1; Santana Pinto de Castro; Katiane Araújo Lourido33; David Chiara4; \\ Kelly Christina Ferreira Castro5; Denise Castro Lustosa ${ }^{6}$. \\ 1 Universidade Federal do Oeste do Pará, Santarém, Pará, Brasil, bruna0909martins@hotmail.com \\ 2 Universidade Federal do Oeste do Pará, Santarém, Pará, Brasil, santanacastro1@hotmail.com \\ 3 Universidade Federal do Oeste do Pará, Santarém, Pará, Brasil, katialourido@gmail.com \\ ${ }^{4}$ Universidade Federal de Itajubá, Itajubá, Minas Gerais, Brasil, davidchiara@gmail.com \\ ${ }^{5}$ Universidade Federal do Oeste do Pará, Santarém, Pará, Brasil, kelly_quimica@yahoo.com.br \\ 6Universidade Federal do Oeste do Pará, Santarém, Pará, Brasil, denise.lustosa@ufopa.edu.br
}

RESUMO: Pertencente à família Verbenaceae, Lippia alba (Mill) N. E. Brown é amplamente utilizada na medicina popular devido suas propriedades analgésicas, calmantes e espasmolíticas. Neste trabalho, buscou-se avaliar o efeito do óleo essencial dessa espécie, nas concentrações de 0,10; 0,25; 0,50; 0,75 e 1,25 $\mu \mathrm{L} \cdot \mathrm{mL}^{-1}$, sobre o crescimento micelial de isolados de Fusarium spp. obtidos de Anacardium occidentale L. (caju); Zea mays L. (milho); Capsicum annuum L. (pimentão); Couratari oblongifolia Ducke \& R. Knuth (tauari) e Rhizoctonia solani obtido da espécie Glycine max L. (soja). No bioensaio, o óleo previamente filtrado foi adicionado em meio BDA (Batata-Dextrose-Ágar), homogeneizado e vertido em placas de Petri. Em seguida, discos contendo estruturas fúngicas foram depositados, centralmente, nas placas com BDA e o óleo em suas respectivas concentrações. O tratamento controle consistiu em placas com meio de cultura sem a presença do óleo. Os dados foram obtidos medindo o diâmetro das colônias fúngicas, durante cinco dias. Todas as concentrações reduziram o crescimento micelial dos fitopatógenos em relação à testemunha, apresentando um percentual de inibição médio de 88,5\%. Para os isolados de Fusarium spp. do caju e do pimentão, bem como Rhizoctonia solani isolado da soja, o óleo apresentou efeito fungicida; sugerindo estudos, in vivo, para constatação de seu efeito fungitóxico, e análises químicas para investigação e isolamento dos compostos ativos da espécie.

PALAVRAS-CHAVE: Controle alternativo, Efeito fungicida, Redução do crescimento.

\section{ESSENTIAL OIL OF LEMONGRASS PATHOGENS ON FOREST AND AGRICULTURAL SPECIES}

ABSTRACT: Belonging to Verbenaceae, Lippia alba (Mill) N. E. Brown family's used in folk medicine because its analgesic properties, calming and spasmolytic. In this work, we tried to evaluate the essential oil effect of this species, in the concentrations of 0.10; 
$0.25 ; 0.50 ; 0.75$ and $1.25 \mu \mathrm{L} . \mathrm{mL}^{-1}$, on the mycelial growth of Fusarium spp. obtained from Anacardium occidentale L. (cashew); Zea mays L. (corn); Capsicum annuum L. (pepper); Couratari oblongifolia Ducke \& R. Knuth (tauari) and Rhizoctonia solani obtained of the species Glycine max L. (soy bean). In the bioassay, the previously filtered oil was added in PDA (Potato-Dextrose-Agar) medium, homogenized and poured into Petri dishes. Then, disks containing fungal structures were deposited, centrally, on the PDA plates and the oil at their respective concentrations. The control treatment consisted of plates with culture medium without the presence of oil. The data were obtained by measuring the diameter of the fungal colonies for five days. All concentrations reduced the mycelial growth of phytopathogens in relation to the control, presenting an average inhibition percentage of $88.5 \%$. For the isolates of Fusarium spp. of cashew and pepper, as well as Rhizoctonia solani isolated from soy bean, the oil presented fungicidal effect; suggesting studies, in vivo, to verify it's fungitoxic effect, and chemical analyzes for investigation and isolation of the active compounds of the species.

KEYWORDS: Alternative control, Fungicidal effect, Reduced growth.

\section{ACEITE ESENCIAL DE HIERBA-CIDERA SOBRE FITOPATÓGENOS DE ESPECIES FORESTALES Y AGRÍCOLAS}

RESUMEN: Pertenencia a la familia Verbenaceae, Lippia alba (Mill) N. E. Brown es ampliamente utilizada en la medicina popular debido a sus propiedades analgésicas, calmantes y espasmolíticas. En este trabajo, se buscó evaluar el efecto aceite esencial de esa especie, en las concentraciones de 0,10;0,25; 0,50; 0,75 y 1,25 $\mathrm{LL}_{\mathrm{mL}} \mathrm{m}^{-1}$, sobre el crecimiento micelial de los aislados de Fusarium spp. obtenidos de Anacardium occidentale L. (cajú); Zea mays L. (maíz); Capsicum annuum L. (chile); Couratari oblongifolia Ducke \& R. Knuth (tauari) y Rhizoctonia solani obtenida de la especie Glycine max L. (soja). En el bioensayo, el aceite previamente filtrado fue agregado en medio BDA (Batata-Dextrosa-Agar), homogeneizado y vertido en placas de Petri. A continuación, los discos que contenían estructuras fúngicas se depositaron centralmente en las placas con BDA y el aceite en sus respectivas concentraciones. El tratamiento control consistió en placas con medio de cultivo sin la presencia del aceite. Los datos fueron obtenidos midiendo el diámetro de las colonias fúngicas, durante cinco días. Todas las concentraciones reducen el crecimiento del micelio de patógenos de plantas en comparación con el control, con un porcentaje de inhibición media de 88,5\%. Para los aislados de Fusarium spp. del cajú y del chile, así como Rhizoctonia solani aislado de la soja, el aceite presentó efecto fungicida; sugiriendo estudios, in 
vivo, para constatacíon de su efecto fungitóxico, y análisis químicos para investigación y aislamiento de los compuestos activos de la especie.

PALABRAS CLAVE: Control alternativo, Efecto fungicida, reducción del crecimiento.

O gênero Lippia pertence à família Verbenaceae e inclui aproximadamente 200 espécies de plantas que possuem distribuição nas regiões tropicais, subtropicais e temperadas da África, Ásia e América (TERBLANCHÉ; KORNELIUS, 1996).

A espécie em estudo, Lippia alba (Mill) N. E. Brown, é originária dos Andes e pode ser encontrada atualmente em todas as regiões tropicais e subtropicais das Américas do Sul e Central, e ilhas do Caribe (LEMOS et al., 1990). É conhecida no Brasil como erva-cidreira, falsamelissa, chá-de-tabuleiro, erva-cidreirado-campo, salva-do-Brasil, salva-limão e erva-cidreira-brava (MATOS, 2000).

Na medicina popular, os extratos e infusões de suas folhas e flores são utilizados para tratamento de doenças pulmonares, febre, problemas digestivos e hepáticos, sífilis, diarreias e disenterias (ALEA et al., 1997; VALE et al., 1999; PASCUAL et al., 2001a).
Estudos sobre a erva-cidreira vêm crescendo na última década, principalmente, os que relacionam aspectos agronômicos como, cultivo e desenvolvimento, localização geográfica, solo e clima, com as características químicas do óleo essencial produzido pela espécie (TAVARES, 2009).

Os constituintes majoritários relatados com maior frequência na literatura para Lippia alba são os quimiotipos contendo citral, carvona e linalol (ATTI-SERAFINI et al., 2002; LORENZI; MATOS, 2002). Ações calmantes e espasmolíticas suaves foram evidenciadas e atribuídas ao citral, enquanto atividade analgésica foi conferida ao mirceno, ambos presentes no óleo essencial (MATOS et al., 1996; ALEA et al., 1997; VIANA et al., 1998).

Segundo Pascual et al. (2001b) e Tavares et al. (2011), outras indicações estão relacionadas à erva-cidreira, 
incluindo as atividades antiprotozoário e antimicrobiana, sendo promissora não apenas para as indústrias farmacêuticas, como também para indústrias de químicos agrícolas, devido aos avanços na utilização de plantas com propriedades antifúngicas, inseticidas e repelentes para fabricação de químicos naturais (YAMAMOTO et al., 2008).

Neste sentido, buscou-se avaliar a atividade antifúngica do óleo essencial de Lippia alba sobre o crescimento micelial de isolados de espécies florestais e agrícolas.

Para a obtenção do óleo essencial, o material vegetal (folhas e galhos) foi triado, seco em temperatura ambiente por 72 horas, pesado e posteriormente armazenado até sua utilização. A extração foi realizada no Laboratório de Pesquisa e Desenvolvimento de Produtos Naturais Bioativos (P\&DBio), da Universidade Federal do Oeste do Pará (UFOPA), por hidrodestilação, em aparelho de Clevenger, com duração de 4 horas. $\bigcirc$ óleo obtido foi acondicionado em frasco âmbar estéril sob refrigeração.
Os fitopatógenos foram obtidos através de materiais vegetais apresentando sintomas de doença, coletados na região, sendo o gênero Fusarium spp. isolado das espécies: Anacardium occidentale L. (Anacardiaceae) - caju; Zea mays L. (Gramineae) - milho; Capsicum annuum L. (Solanaceae) - pimentão e Couratari oblongifolia Ducke \& R. Knuth (Lecythidaceae) - tauari, e Rhizoctonia solani isolado da espécie Glycine max L. (Fabaceae) - soja. Os fungos encontram-se armazenados na micoteca do Laboratório de Fitopatologia, do Instituto de Biodiversidade e Florestas (IBEF), na Universidade Federal do Oeste do Pará (UFOPA), Santarém-PA.

O ensaio biológico foi desenvolvido no Laboratório de Fitopatologia, da UFOPA, Santarém-PA.

O óleo de Lippia alba foi previamente filtrado em Millipore de porosidade 0,46 $\mu \mathrm{m}$ e adicionado em meio BDA (BatataDextrose-Ágar), fundente $\left(\cong 45^{\circ} \mathrm{C}\right)$, para a obtenção das concentrações de 0,10; 0,$25 ; 0,50 ; 0,75$ e $1,25 \mu \mathrm{L} \cdot \mathrm{mL}^{-1}$. Em 
seguida, o meio de cultura contendo o óleo, nas respectivas concentrações, foi homogeneizado e vertido em placas de Petri. Após solidificação do BDA, depositou-se centralmente, em todas as placas do ensaio, um disco de meio $(0,4$ $\mathrm{cm}$ ) contendo as estruturas dos fitopatógenos, que foram incubados a $25^{\circ} \mathrm{C}$, sob fotoperíodo de 12 horas.

O tratamento controle consistiu do cultivo dos fungos em meio BDA, sem a adição de óleo. O delineamento experimental foi inteiramente casualizado (DIC), em esquema fatorial, com quatro repetições.

Avaliou-se o diâmetro médio das colônias, em intervalos de 24 horas, durante cinco dias. Os dados obtidos foram comparados pelo Teste de Tukey a $5 \%$ de probabilidade e analisados pelo software estatístico Assistat ${ }^{\circledR} 7.7$ beta (SILVA, 2013).

Os resultados obtidos foram significativos tanto para os fatores isolados (concentrações e fitopatógenos), quanto para a interação entre eles $(p<0,01)$.
Verificou-se que a redução do crescimento micelial dos fitopatógenos ocorreu de forma linear ao aumento das concentrações do óleo (Figura 1). $\mathrm{Na}$ concentração de $0,10 \mu \mathrm{L} \cdot \mathrm{mL}^{-1}$, essa redução do crescimento já representava 11,3\% em relação ao tratamento controle. A maior concentração em teste $\left(1,25 \mu \mathrm{L} . \mathrm{mL}^{-1}\right)$, proporcionou um percentual de inibição médio de 88,5\%.

Schwan-Estrada et al. (2000), apontaram em seus estudos o potencial da erva-cidreira no controle de fungos fitopatogênicos. Rao et al. (2000), constataram a superioridade do seu efeito em relação a fungicidas comerciais, e indicaram a possibilidade da aplicação dessa espécie como defensivo agrícola.

Quanto ao fator fitopatógenos, todos os isolados obtiveram médias menores que $4,3 \mathrm{~cm}$. $O$ gênero Fusarium spp. isolado do tauari obteve a maior redução no crescimento micelial $(3,6 \mathrm{~cm})$, seguido dos isolados obtidos do pimentão, do pseudofruto do cajueiro e de Rhizoctonia solani, estes, não diferiram estatisticamente 
entre si e apresentaram uma média de crescimento de 3,9 cm. O Fusarium sp. isolado do milho apresentou uma maior resistência à ação do óleo (diâmetro médio de 4,2 cm), porém não diferiu estatisticamente dos isolados mencionados anteriormente (Figura 2).

Dubey et al. (1983) também observaram efeito fungitóxico de folhas de erva-cidreira sobre o fungo de solo R. solani. Brand et al. (2006), constataram que extratos brutos de erva-cidreira inibiram o crescimento micelial de Sclerotinia sclerotiorum em 43,9\%. Ferreira et al. (2014), destacaram a erva-cidreira no controle alternativo, in vitro, de Colletotrichum gloeosporioides, fungo causador da antracnose em frutos de mamão.

Avaliando a interação concentrações e fitopatógenos, verificou-se que para o gênero Fusarium spp. isolado do caju e do pimentão, a concentração de 1,25 $\mu \mathrm{L} . \mathrm{mL}^{-1}$ do óleo ocasionou efeito fungicida. Este efeito foi observado desde a concentração de 0,50 $\mu \mathrm{L} \cdot \mathrm{mL}^{-1}$ até a concentração de 1,25 $\mu \mathrm{L} \cdot \mathrm{mL}^{-1}$ para R. solani (Tabela 1).

A concentração de 1,25 $\mu \mathrm{L} \cdot \mathrm{mL}^{-1}$ também ocasionou as maiores reduções para Fusarium spp. do milho, com percentual de inibição de 78,6\% em relação ao controle, e para o isolado do tauari, com percentual de 79,4\%, não diferindo estatisticamente da concentração de 0,75 $\mu \mathrm{L} \cdot \mathrm{mL}^{-1}$.

Tereschuk et al. (1997), relataram que o extrato bruto de L. alba possui ação fungitóxica, in vitro, sobre Alternaria alternata, Colletotrichum graminicola, $R$. solani e Sclerotium rolfsii. Vicenço et al. (2017), avaliando o efeito desse óleo sobre Cercorpora kikuchii, constataram uma inibição de 100\% a partir da concentração em teste de 0,15\%.

O óleo de Lippia alba, nas maiores contrentrações, apresentou efeito fungicida para a maioria dos fitopatógenos utilizados no trabalho. 
Figura 1. Efeito fungitóxico do óleo de erva-cidreira, nas diferentes concentrações de teste, determinado através do Diâmetro Médio das Colônias (cm).

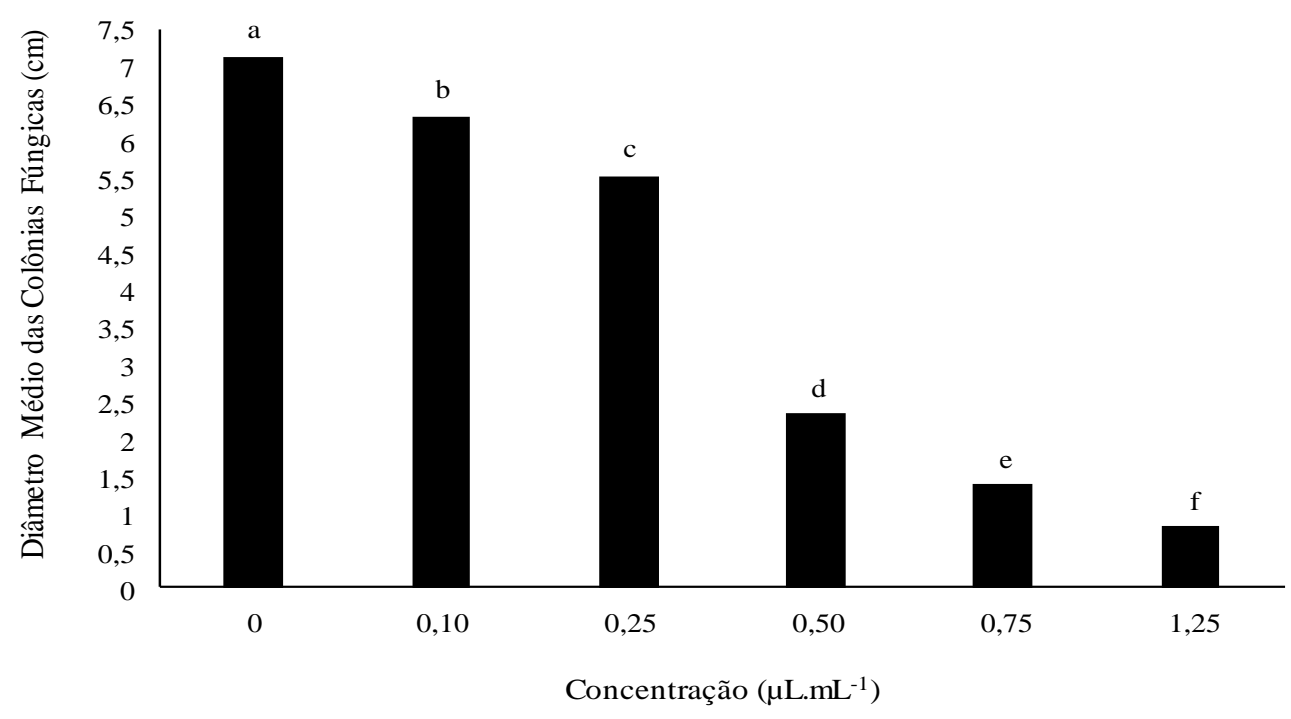

Figura 2. Efeito fungitóxico do óleo de erva-cidreira sobre os diferentes fitopatógenos, determinado através do Diâmetro Médio das Colônias (cm).

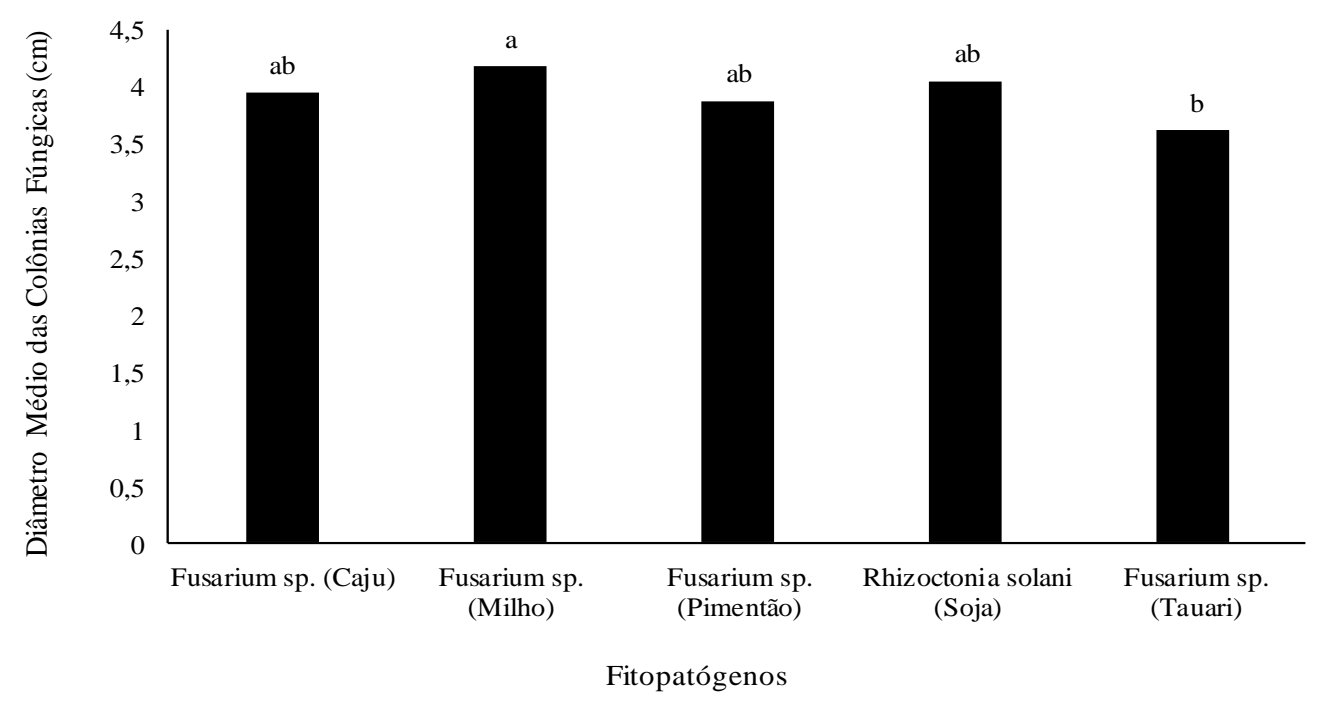


Tabela 1. Atividade antifúngica do óleo de erva-cidreira, em diferentes concentrações, sobre os fitopatógenos confrontados.

\begin{tabular}{cccccc}
\hline \multicolumn{5}{c}{ Diâmetro Médio das Colônias (cm) } \\
\hline $\begin{array}{c}\text { Concentrações } \\
\left(\mu \mathrm{L} . \mathrm{mL}^{-1}\right)\end{array}$ & $\begin{array}{c}\text { Fusarium } \\
\text { sp. (Caju) }\end{array}$ & $\begin{array}{c}\text { Fusarium sp. } \\
\text { (Milho) }\end{array}$ & $\begin{array}{c}\text { Fusarium sp. } \\
\text { (Pimentão) }\end{array}$ & $\begin{array}{c}\text { Rhizoctonia } \\
\text { solani (Soja) }\end{array}$ & $\begin{array}{c}\text { Fusarium sp. } \\
\text { (Tauari) }\end{array}$ \\
\hline 0 & $7,0 \mathrm{aB}$ & $7,0 \mathrm{aB}$ & $6,8 \mathrm{aB}$ & $8,6 \mathrm{aA}$ & $6,3 \mathrm{aB}$ \\
0,10 & $5,7 \mathrm{bB}$ & $6,1 \mathrm{abB}$ & $6,2 \mathrm{abB}$ & $8,3 \mathrm{aA}$ & $5,4 \mathrm{aB}$ \\
0,25 & $5,2 \mathrm{bA}$ & $5,9 \mathrm{bA}$ & $5,2 \mathrm{bA}$ & $6,2 \mathrm{bA}$ & $5,2 \mathrm{aA}$ \\
0,50 & $3,5 \mathrm{cA}$ & $2,5 \mathrm{cAB}$ & $3,0 \mathrm{cAB}$ & $0,4 \mathrm{cC}$ & $2,2 \mathrm{bB}$ \\
0,75 & $1,8 \mathrm{dA}$ & $2,0 \mathrm{cA}$ & $1,5 \mathrm{dAB}$ & $0,4 \mathrm{cB}$ & $1,3 \mathrm{bAB}$ \\
1,25 & $0,4 \mathrm{eA}$ & $1,5 \mathrm{cA}$ & $0,5 \mathrm{dA}$ & $0,4 \mathrm{cA}$ & $1,3 \mathrm{bA}$ \\
\hline CV\% & & & & 14,63
\end{tabular}

*Médias seguidas pelas mesmas letras minúsculas nas colunas e pelas mesmas letras maiúsculas nas linhas não diferem entre si pelo teste de Tukey a $5 \%$ de probabilidade.

\section{REFERÊNCIAS}

ALEA, J. A. P.; LUIS, A. G. O.; PÉREZ, A. R.; JORGE, M. R.; BALUJA, R. Composición y propiedades antibacterianas del aceite es- sencial de Lippia alba (Mill.) N. E. Brown. Revista Cubana de Farmácia, v.30, p.1 - 6, 1997.

ATTI-SERAFINI, L.; PANCERA, M. R.; ATTI, S. A. C.; ROSSATO, M.; PAULETTI, G. F.; ROTAL, D.; PAROOUL, N.; MOYNA, P. Variation in essential oil
CONGRESSO DE INICIAÇÃO CIENTÍFICA, 2006, Pelotas. Resumos... Pelotas: Universidade Federal de Pelotas, 2006.

DUBEY, N. K.; KISHORE, N; SRIVASTAVA, O. P.; DIKSHIT, A.; SINGH, S. K. Fungitoxicity of some higher plants against Rhizoctonia solani. Plant and Soil, v.72, p.91 - 94, 1983.

FERREIRA, E. F.; JOSÉ, A. R. S.; BOMFIM,

*Médias seguidas pelas mesmas letras minúsculas nas colunas e pelas mesmas letras maiúsculas nas linhas não diferem entre si pelo teste de Tukey a $5 \%$ de probabilidade.

Revista Brasileira de Plantas Medicinais, v.4, p.72- 74, 2002.

BRAND, S. C.; JUNGES, E.; MILANESI, P.; BLUME, E.; MUNIZ, M. F. B. Extratos vegetais aquosos no crescimento micelial de Sclerotinia sclerotiorum. In:
Colletotrichum gloeosporioides Penz. coletado em frutos de mamoeiro (Carica papaya L.). Revista Brasileira de Fruticultura, v.36, n.2, p.346 - 352, 2014.

LEMOS, T. L. G.; MATOS, F. J. A.; ALENCAR, J. W.; CRAVEIRO, A. A.; 
MCCHESNEY, J. D. Antimicrobial activity of essential oil of Brazilian plants. Phytotherapy Research, V.4, p.82 - 84, 1990.

LORENZI, H.; MATOS, F. J. A. Plantas medicinais do Brasil: nativas e exóticas cultivadas. Nova Odessa-SP: Instituto Plantarum, 2002. 512 p.

MATOS, F. J. A. Plantas Medicinais: guia de seleção e emprego de plantas usadas em fitoterapia no Nordeste do Brasil. 2. ed. Fortaleza: Imprensa Universitária, 2000. 346 p.

MATOS, F. J. A.; MACHADO, M. I. L.; CRAVEIRO, A. A.; ALENCAR, J. W. The essential oil composition of two chemotypes of Lippia alba grown in Northeast Brazil. Journal of Essential Oil Research, v.8, n.6, p.695 - 698, 1996.

PASCUAL, E. M.; SLOWING, K.; CARRETERO, M. E.; VILLAR, A. Antiulcerogenic activity of Lippia alba (Mill.) N. E. Brown (Verbenaceae). II Farmaco, n.56, p.501 - 504, 2001a.

PASCUAL, M. E.; SLOWING, K.; CARRETERO, E. et al. Lippia: traditional uses, chemistry and pharmacology: a review. Journal of Ethnopharmacology, v.76, p.201 - 214, 2001b.

RAO, G. P.; SINGH, M.; SINGH, P.; SINGH, S. P.; CATALAN, C.; KAPOOR, I. P. S.; SINGH, O. P.; SINGH, G. Studies on chemical constituents and antifungal activity of leaf essential oil of Lippia alba (Mill.). Indian Journal of Chemical Technology, v.7, p.332 - 335, 2000.
SCHWAN-ESTRADA, K. R. F.; STANGARLIN, J. R.; CRUZ, M. E. R. Uso de extratos vegetais no controle de fungos fitopatogênicos. Floresta, v.30, p.129 - 137, 2000.

SILVA, F. A. S. ASSISTAT Versão 7.7 beta. Universidade Federal de Campina Grande, Campina Grande, Paraíba, 2013. Disponível em: <http://www.assistat.com>. Acesso: 20 out. 2017.

TAVARES, I. B. Propagação vegetativa, adubação orgânica e idades de colheita de quimiotipos de ervacidreira [Lippia alba (Mill.) N. E. Brown], Gurupi, 2009. 84 f. Dissertação (Mestrado - Área de Concentração em Produção Vegetal) - Departamento de Produção Vegetal, Universidade Federal do Tocantins, Gurupi, 2009.

TAVARES, I. B.; MOMENTÉ, V. G.; NASCIMENTO, I. R. do. Lippia alba: estudos químicos, etnofarmacológicos e agronômicos. Revista Brasileira de Tecnologia Aplicada nas Ciências Agrárias, v.4, n.1, p.204 - 220, 2011.

TERBLANCHÉ, F. C.; KORNELIUS, G. Essential oil constituintes of the genus Lippia (Verbenaceae): a literature review. Journal of Essential Oil Research, v.8, p.471 - 485, 1996.

TERESCHUK, M. L.; RIEIRA, M. V. Q.; CASTRO G. R.; ABDALA L. R. Antimicrobial activity offlavonoids from leaves of Tagetes minuta. Journal of Ethnopharmacology, v.56, p.227 - 232, 1997. 
VALE, T. G.; MATOS, F. J. A.; LIMA, T. C. M.; VIANA, G. S. B. Behavioral effects of essential oils from Lippia alba (Mill.) N. E. BROWN chemotypes. Journal of Ethnopharmacology, v.167, n.1, p.127 133, 1999.

VIANA, G. S. B.; VALE, T. G.; RAO, V. S. N.; MATOS, F. J. A. Analgesic and antiinflamatory effects of two chemotypes of Lippia alba: a comparative study. Pharmaceutical Biology, v.36, n.4, p.1 - 5, 1998.

VICENÇO, C. B.; FELTRACCO, M. C.; CAVIÃO, H. C.; PANSERA, M. R.; SARTORI, V. C. Composição química e atividade in vitro de óleos essenciais sobre a mancha púrpura da soja. Revista Interdisciplinar de Ciência Aplicada, v.2, n.4, 2017.

YAMAMOTO, P. Y; COLOMBO, C. A; AZEVEDO FILHO, J. A; LOURENÇÃO, A. L; MARQUES, M. O. M; MORAIS, G. D. S; CHIORATO, A. F; MARTINS, A. L. M.; SIQUEIRA, W. J. Performance of ginger Grass (Lippia alba) for traits related to the production of essencial oil. Scientia Agrícola, v.65, n.5, p.481 - 489, 2008. 